\title{
State-Level Emergency Preparedness and Response Capabilities
}

\author{
Sharon M. Watkins, PhD; Dennis M. Perrotta, PhD; Martha Stanbury, MSPH; \\ Michael Heumann, MPH, MA; Henry Anderson, MD; Erin Simms, MPH; Monica Huang, MPH
}

\section{ABSTRACT}

Background: Prior assessments of public health readiness had identified gaps in radiation preparedness. In recent years, preparedness planning has involved an "all-hazards" approach. Current assessment of the national status related to radiation public health emergency preparedness capabilities at the state and local health department levels was needed.

Methods: A survey of state health departments related to radiation readiness was undertaken in 2010 by the Council of State and Territorial Epidemiologists (CSTE). States with nuclear power plants were instructed to consider their responses exclusive of capabilities and resources related to the plants given that the emergency response plans for nuclear power plants are specific and unique.

Results: Thirty-eight (76\%) state health departments responded to the survey, including 26 of the 31 states with nuclear power plants. Specific strengths noted at the state level included that the majority of states had a written radiation response plan and most plans include a detailed section for communications issues during a radiation emergency. In addition, more than half of the states indicated that their relationship with federal partners is sufficient to provide resources for radiation emergencies, indicating the importance states placed on federal resources and expertise. Specific weaknesses are discussed and include that most states had completed little to no planning for public health surveillance to assess potential human health impacts of a radiation event; less than half had written plans to address exposure assessment, environmental sampling, human specimen collection and analysis, and human health assessment. Few reported having sufficient resources to do public health surveillance, radiation exposure assessment, laboratory functions and other capabilities.

Discussion: Levels of planning, resources and partnerships varied among states, those with nuclear power plants were better prepared. Gaps were evident in all states; however and additional training and resources are needed to ensure adequate levels of preparedness.

Conclusion: Overall results of this assessment indicate that in most measures of public health capacity and capability, states are poorly prepared to adequately respond to a major radiation emergency event. Specific recommendations are noted in the discussion.

(Disaster Med Public Health Preparedness. 2011;5:S134-S142)

Key Words: radiation, emergency preparedness, state-level capabilities, disaster, radiation preparedness planning

1 ttention on public health preparedness has increased since the September 11, 2001, terrorist attacks on New York City's World Trade Center and other sites. The Council of State and Territorial Epidemiologists (CSTE) conducted a national assessment of the status of planning for public health preparedness for chemical and radiation terrorism in $2003^{1}$ and identified substantial gaps in preparedness and response capabilities. In recent years, preparedness planning has expanded to an all-hazards approach that includes readiness to respond not only to terrorism but also to releases from unintentional technological incidents, natural disasters, and outbreaks of human diseases.

Guidance for emergency preparedness planning and response activities and capabilities related to radiation release incidents (both intentional and unintentional) has come from a collaborative focus among the Centers for Disease Control and Prevention (CDC), the Conference of Radiation Control Program Directors, the Association of State and Territorial Health Officials, the Association of Public Health Laboratories, the National Association of County and City Health Officials, and CSTE. These organizations and agencies have formed the National Alliance for Radiation Readiness (NARR) to increase awareness and understanding of the varied public health responsibilities related to radiation emergencies and to improve communication across the divergent communities responsible for preparedness and response.

As part of the initial activities undertaken by NARR, CSTE reassessed the national status of radiation preparedness planning and capabilities at the state health department level, which encompassed radiologic ter- 
rorism and unintentional and natural radiation releases that could harm human health. Because states that have nuclear power-generating plants already have established and exercised radiation emergency response capabilities, this assessment characterized capabilities independent of nuclear power plant operations. The purpose of this assessment was to identify gaps in planning and response capabilities at state health agencies which could be used to inform national partners and enable targeting of additional resources and efforts to improve the nation's overall preparedness and response capabilities regarding radiation emergencies. (The full report can be found at the CSTE Web site, www.cste.org.)

\section{METHODS}

The 2003 CSTE chemical and radiologic terrorism assessment was used as the starting point for the current survey, and questions were added to broaden the scope to include details about preparedness activities for all radiation emergencies, not just terrorism. The 2010 assessment instrument was changed enough from the 2003 instrument to make direct comparisons difficult.

The new draft assessment was created by a CSTE workgroup composed of epidemiologists from 4 state health departments, CSTE staff, and a consultant epidemiologist. It was further developed after review by other agencies and organizations of NARR. The instrument was pilot tested in 3 state health departments, and suggestions were incorporated into the final version. The assessment was adapted for electronic completion using SurveyMonkey (SurveyMonkey, Palo Alto, CA).

The questionnaire was divided into 4 categories: radiation emergency preparedness and response capability (planning and resources); radiation emergency staffing levels in state health departments; local relationships; and interagency/intra-agency coordination on radiation emergency preparedness and response activities. This article focuses on findings related to preparedness and response capability, staffing, and interagency coordination.

Within the first category, preparedness and response capability (planning and resources), there were 4 discrete groups: planning, available resources internally, available resources in other state agencies, and relationships with federal agencies. Within each group, questions addressed epidemiology and surveillance, exposure assessment, handling of environmental and biological samples, radiological health assessment, and other public health functions (including communications, potassium iodide, and worker health and safety consultations). Following this, questions addressed overall radiological emergency plans and exercises and the extent of planning for specific radiological release scenarios: the release type (unintentional vs intentional) and the environmental situation in which the release occurs (transportation, medical facility, mass gathering, major location, others).
For the category radiation emergency staffing levels in state health departments, states were asked to estimate the number of staff members available for response in an event and to categorize them by funding source.

Findings for the third category of the survey, local relationships, were not substantially different from findings related to other relationships presented (agency level, federal level), and are not detailed in this article.

For the fourth category, interagency/intra-agency coordination on radiation emergency preparedness and response activities, states were asked to report on the level of coordination for radiation emergency preparedness and response activities between their agency and a variety of other agencies and institutions, with the level of coordination ranging from noncontact to exercise conducted within the last 2 years. States were asked to note whether they had written memoranda of understanding with any of the listed partner agencies or organizations. State public health agencies also were asked to report whether their radiation response team meets with other public health divisions (eg, laboratory, epidemiology) to coordinate responses to radiation emergency incidents. States were asked whether they had developed planning and response protocols for gathering epidemiologic and exposure data and for providing coordinated guidance for large-scale radiation emergency incidents that would involve more than 1 county in the state.

State epidemiologists in all 50 states were asked to complete the assessment electronically using the best information they could obtain from their health agency, including seeking input of the most qualified and involved agency staff from radiation, epidemiology, and laboratory services. They were asked to note whether their state's official radiation control program director participated in preparing their responses. All of the questions, unless otherwise noted, explicitly excluded capabilities directly related to nuclear power plant emergency response. Most of the questions asked states to rate their planning, resources, and relationships using a 4- or 5-choice rating system that ranged from none to sufficient number and level. Finally, each state was asked to provide an overall rating of their public health agency's preparedness to respond to a major radiation emergency incident, choosing a number on a scale of 0 (not prepared at all) to 10 (fully prepared).

Data were analyzed by CSTE using SAS (SAS Institute, Cary, $\mathrm{NC)}$; calculated frequencies and descriptive statistics are provided as aggregated data so that responses for individual states or territories are not identifiable. (The survey instrument is available at the CSTE Web site: http://www.cste.org/webpdfs /2010raditionreport.pdf.)

\section{RESULTS}

Thirty-eight (76\%) states responded to the questionnaire, but not all responding states answered all of the questions. Responding states varied by size, population, region, and presence of an operating nuclear power plant within their borders. 
Of the 31 states with nuclear power plants, 26 (84\%) responded to the assessment. Twelve (63\%) of the 19 states without nuclear power plants completed the assessment.

\section{Planning and Resources Assessment Planning}

The extent of planning for epidemiology and surveillance for the human health effects of radiation was assessed for 5 types: syndromic surveillance, clinician reporting, crisis-phase epidemiology, recovery-phase epidemiology, and other types of statistical surveillance (Table 1). A range between 70\% and 84\% of states reported minimal to no planning completed on the potential human effects of radiation among any of these 5 types of surveillance.

States reported only slightly better planning for providing advice on exposure assessment and environmental sampling com- bined (42\%-50\% reporting none to minimal planning) and little planning to provide advice for biological sampling ( $14 \%$ have none and $60 \%$ have minimal). Seventy-four percent of states reported having minimal $(53 \%)$ or no $(21 \%)$ plans to conduct population-based exposure monitoring.

Thirty-one (82\%) states reported no or minimal plans to collect biological or clinical samples, and $28(74 \%)$ indicated no or minimal plans for processing and shipping samples for radioactivity analysis. Only 5 (13\%) states reported having any written or detailed operations plan for radiologic analyses of biological or clinical samples. More states reported having a written plan or detailed operations plan for addressing collection (20 states [54\%]), processing (17 [46\%]), shipping (14 [38\%]) of environmental samples and conducting (14 [38\%]) radioactivity analysis than they did plans addressing biological/ clinical samples.

\section{TABLE 1}

\section{Extent of Planning in State Health Departments for Radiation Emergencies}

\begin{tabular}{|c|c|c|c|c|}
\hline & $\begin{array}{l}\text { None, } \\
\text { No. }(\%)\end{array}$ & $\begin{array}{l}\text { Minimal, } \\
\text { No. (\%) }\end{array}$ & $\begin{array}{l}\text { Written Plan, } \\
\text { No. (\%) }\end{array}$ & $\begin{array}{c}\text { Detailed } \\
\text { Operations } \\
\text { Plan, No. (\%) }\end{array}$ \\
\hline Syndromic surveillance related to radiation incident & $13(34.2)$ & $18(47.4)$ & $4(10.5)$ & $3(7.9)$ \\
\hline $\begin{array}{l}\text { Other kind of surveillance (eg, poison control center calls, pharmaceutical } \\
\text { purchases, school absenteeism), not included above }\end{array}$ & $8(21.1)$ & $20(52.6)$ & $6(15.8)$ & $4(10.5)$ \\
\hline $\begin{array}{l}\text { Recovery-phase epidemiology (eg, documenting delayed health effects, exposure } \\
\text { registries) }\end{array}$ & $10(27.0)$ & $21(56.8)$ & $3(8.1)$ & $3(8.1)$ \\
\hline \multicolumn{5}{|l|}{ Exposure assessment } \\
\hline Advice for radiation exposure assessment & $2(5.3)$ & $14(36.8)$ & $13(34.2)$ & $9(23.7)$ \\
\hline Advice regarding environmental sampling & $6(15.8)$ & $13(34.2)$ & $11(28.9)$ & $8(21.1)$ \\
\hline Advice regarding biological/clinical sampling & $5(13.5)$ & $22(59.5)$ & $7(18.9)$ & $3(8.1)$ \\
\hline Ability to ship for radioactivity analysis & $10(27.0)$ & $13(35.1)$ & $9(24.3)$ & $5(13.5)$ \\
\hline Ability to conduct radioactivity analysis & $10(27.0)$ & $13(35.1)$ & $5(13.5)$ & $9(24.3)$ \\
\hline \multicolumn{5}{|l|}{ For biological/clinical samples } \\
\hline Ability to collect for radioactivity analysis & $10(26.3)$ & $21(55.3)$ & $4(10.5)$ & $3(7.9)$ \\
\hline Ability to process for radioactivity analysis & $14(37.8)$ & $14(37.8)$ & $5(13.5)$ & $4(10.8)$ \\
\hline Ability to ship for radioactivity analysis & $10(26.3)$ & $18(47.4)$ & $6(15.8)$ & $4(10.5)$ \\
\hline Ability to conduct radioactivity analysis & $17(45.9)$ & $15(40.5)$ & $3(8.1)$ & $2(5.4)$ \\
\hline \multicolumn{5}{|l|}{ Health assessment } \\
\hline Health physics interpretation of acute incident-radiation & $7(18.4)$ & $17(44.7)$ & $9(23.7)$ & $5(13.2)$ \\
\hline Health physics consultation on reentry-radiation & $11(28.9)$ & $7(18.4)$ & $15(39.5)$ & $5(13.2)$ \\
\hline Health physics predictions on long-term health effects-radiation & $13(34.2)$ & $12(31.6)$ & $8(21.1)$ & $5(13.2)$ \\
\hline Medicine consults regarding radiation effects & $9(24.3)$ & $17(45.9)$ & $8(21.6)$ & $3(8.1)$ \\
\hline Early detection of radiation contamination in first responders & $6(15.8)$ & $10(26.3)$ & $12(31.6)$ & $10(26.3)$ \\
\hline
\end{tabular}

IT/GIS= information technology/geographic information systems. 
No or minimal health physics interpretation, predictions planning, and planning for medicine consultations were reported by $63 \%$ to $70 \%$ of states. Sixteen (42\%) states reported minimal or no planning to detect radiation contamination in first responders. Likewise, 25 states (66\%) reported minimal to no planning to provide health physics predictions on the longterm health effects of radiation. Eight (21\%) states reported planning for information technology/geographic information systems integration of radiation exposure data.

A total of $58 \%$ to $68 \%$ of states reported having written plans or detailed operations plans for health alerts, risk communication, and public communication. Seventy-three percent reported written or detailed operations plans for potassium iodide or other radiation drug-dispensing activity. Half of the states reported having written or detailed operations plans for worker safety/safety consultations.

\section{Resources Within the Agency}

A substantial number of states reported having inadequate resources within their state health department to maintain radiation emergency preparedness (responses include none, none dedicated, some dedicated [Table 2]). No more than 4 (11\%) states reported having sufficient resources for any of the epidemiologic functions associated with a radiation incident.

A total of $76 \%$ to $86 \%$ of states reported having few resources to provide advice for radiation exposure assessments, environmental sampling, and biological/clinical sampling. Only 3 (8\%) states reported adequate resources to conduct populationbased exposure monitoring.

A total of $83 \%$ to $89 \%$ reported insufficient resources to collect, process, and ship samples for and conduct radioactivity analyses of environmental samples. States reported similarly for

\section{TABLE 2}

\section{Resources Available for Radiation Emergencies in State Health Departments}

\begin{tabular}{|c|c|c|c|c|c|}
\hline & $\begin{array}{l}\text { None, } \\
\text { No. }(\%)\end{array}$ & $\begin{array}{c}\text { None } \\
\text { Dedicated, } \\
\text { No. (\%) }\end{array}$ & $\begin{array}{c}\text { Some } \\
\text { Dedicated, } \\
\text { No. (\%) }\end{array}$ & $\begin{array}{c}\text { Sufficient No. } \\
\text { and Level, } \\
\text { No. (\%) }\end{array}$ & $\begin{array}{l}\text { Uncertain, } \\
\text { No. }(\%)\end{array}$ \\
\hline Syndromic surveillance related to radiation incident & $3(7.9)$ & $17(44.7)$ & $15(39.5)$ & $2(5.3)$ & $1(2.6)$ \\
\hline $\begin{array}{l}\text { Other type of surveillance (eg, poison control center calls, } \\
\text { pharmaceutical purchases, school absenteeism), not included above }\end{array}$ & $2(5.3)$ & $19(50.0)$ & $14(36.8)$ & $2(5.3)$ & $1(2.6)$ \\
\hline Surveillance through astute health care providers' reporting & $5(13.5)$ & $13(35.1)$ & $13(35.1)$ & $4(10.8)$ & $2(5.4)$ \\
\hline $\begin{array}{l}\text { Recovery-phase epidemiology (eg, documenting delayed health effects, } \\
\text { exposure registries) }\end{array}$ & $6(16.2)$ & $17(45.9)$ & $10(27.0)$ & $2(5.4)$ & $2(5.4)$ \\
\hline \multicolumn{6}{|l|}{ Exposure assessment } \\
\hline Advice for radiation exposure assessment & $1(2.6)$ & $11(28.9)$ & $17(44.7)$ & $9(23.7)$ & 0 \\
\hline Advice regarding environmental sampling & $3(8.1)$ & $9(24.3)$ & $17(45.9)$ & $8(21.6)$ & 0 \\
\hline Advice regarding biological/clinical sampling & $4(10.8)$ & $15(40.5)$ & $13(35.1)$ & $4(10.8)$ & $1(2.7)$ \\
\hline Ability to ship for radioactivity analysis & $6(16.7)$ & $9(25.0)$ & $17(47.2)$ & $3(8.3)$ & $1(2.8)$ \\
\hline Ability to conduct radioactivity analysis & $8(22.2)$ & $5(13.9)$ & $17(47.2)$ & $5(13.9)$ & $1(2.8)$ \\
\hline \multicolumn{6}{|l|}{ For biological/clinical samples } \\
\hline Ability to collect for radioactivity analysis & 7 (18.4) & $20(52.6)$ & $8(21.1)$ & $2(5.3)$ & $1(2.6)$ \\
\hline Ability to process for radioactivity analysis & $11(29.7)$ & $12(32.4)$ & $9(24.3)$ & $4(10.8)$ & $1(2.7)$ \\
\hline Ability to ship for radioactivity analysis & $8(21.1)$ & $17(44.7)$ & $8(21.1)$ & $4(10.5)$ & $1(2.6)$ \\
\hline Ability to conduct radioactivity analysis & $13(35.1)$ & $13(35.1)$ & $7(18.9)$ & $3(8.1)$ & $1(2.7)$ \\
\hline \multicolumn{6}{|l|}{ Health assessment } \\
\hline Health physics interpretation of acute incident-radiation & $5(13.2)$ & $14(36.8)$ & $15(39.5)$ & $4(10.5)$ & 0 \\
\hline Health physics consultation on reentry-radiation & $5(13.2)$ & $12(31.6)$ & $17(44.7)$ & $4(10.5)$ & 0 \\
\hline Health physics predictions about long-term health effects-radiation & $6(15.8)$ & $13(34.2)$ & $15(39.5)$ & $2(5.3)$ & $2(5.3)$ \\
\hline Medicine consults regarding radiation effects & $6(15.8)$ & $20(52.6)$ & $8(21.1)$ & $3(7.9)$ & $1(2.6)$ \\
\hline Early detection of radiation contamination in first responders & $5(13.5)$ & $15(40.5)$ & $10(27.0)$ & $7(18.9)$ & 0 \\
\hline
\end{tabular}

IT/GIS=information technology/geographic information systems. 
biological/clinical samples, with $86 \%$ to $92 \%$ reporting insufficient resources to collect, process, and ship samples for, and conduct, radiation analyses.

No more than 4 (11\%) state health departments reported having sufficient resources to provide health physics interpretations, consultations upon reentry, predictions on long-term health effects, and medical consultations on radiation effects. Seven (19\%) states reported sufficient resources for early detection of radiation contamination in first responders. A total of $74 \%$ to $84 \%$ of states reported insufficient resources for any of the following: worker health/safety consultations, health alerts, potassium iodide plans, risk communication, and community relations.

\section{Resources Available From Other State Agencies}

As shown in Table 3, a total of $67 \%$ to $86 \%$ of states reported that agencies other than health departments had less than suf- ficient resources for epidemiology/surveillance, exposure assessments, environmental samples handling, biological/clinical samples handling, health assessments, and other public health functions. Notably, up to 9 (24\%) states in each functional category were uncertain about the resources that existed in other state agencies for radiation emergency preparedness.

\section{Relationships With Federal Agencies for Radiation Emergencies}

Substantial resources and capacity for radiation emergency preparedness are located within a variety of federal agencies and are potentially available to states that have established relationships with these agencies (Table 4). Relationships regarding the functions of epidemiology and surveillance varied by specific function but were assessed as sufficient in 16\% to $27 \%$ of states. Relationships with federal partners regarding exposure assessments, handling of environmental samples, health

\section{TABLE 3}

\section{Resources Available for Radiation Emergencies in Other State Agencies}

\begin{tabular}{|c|c|c|c|c|c|}
\hline & $\begin{array}{l}\text { None, } \\
\text { No. }(\%)\end{array}$ & $\begin{array}{c}\text { None } \\
\text { Dedicated, } \\
\text { No. }(\%)\end{array}$ & $\begin{array}{c}\text { Some } \\
\text { Dedicated, } \\
\text { No. (\%) }\end{array}$ & $\begin{array}{l}\text { Sufficient No. } \\
\text { and Level, } \\
\text { No. (\%) }\end{array}$ & $\begin{array}{l}\text { Uncertain } \\
\text { No. (\%) }\end{array}$ \\
\hline Syndromic surveillance related to radiation incident & $16(42.1)$ & $8(21.1)$ & $7(18.4)$ & $1(2.6)$ & $6(15.8)$ \\
\hline $\begin{array}{l}\text { Other kind of surveillance (eg, poison control center calls, } \\
\text { pharmaceutical purchases, school absenteeism), not included above }\end{array}$ & $14(36.8)$ & $6(15.8)$ & $8(21.1)$ & $2(5.3)$ & $8(21.1)$ \\
\hline Surveillance through astute health care providers' reporting & $14(37.8)$ & $7(18.9)$ & $5(13.5)$ & $2(5.4)$ & $9(24.3)$ \\
\hline $\begin{array}{l}\text { Recovery-phase epidemiology (eg, documenting delayed health effects, } \\
\text { exposure registries) }\end{array}$ & $17(45.9)$ & $7(18.9)$ & $3(8.1)$ & $1(2.7)$ & $9(24.3)$ \\
\hline \multicolumn{6}{|l|}{ Exposure assessment } \\
\hline Advice for radiation exposure assessment & $3(8.1)$ & $7(18.9)$ & $16(43.2)$ & $6(16.2)$ & $5(13.5)$ \\
\hline Advice regarding environmental sampling & $3(8.1)$ & $8(21.6)$ & $17(45.9)$ & $5(13.5)$ & $4(10.8)$ \\
\hline Advice regarding biological/clinical sampling & $8(22.2)$ & $9(25.0)$ & $11(30.6)$ & $3(8.3)$ & $5(13.9)$ \\
\hline Ability to ship for radioactivity analysis & $4(11.4)$ & $8(22.9)$ & $12(34.3)$ & $4(11.4)$ & $7(20.0)$ \\
\hline Ability to conduct radioactivity analysis & $9(25.7)$ & $6(17.1)$ & $11(31.4)$ & $4(11.4)$ & $5(14.3)$ \\
\hline \multicolumn{6}{|l|}{ For biological/clinical samples } \\
\hline Ability to collect for radioactivity analysis & $14(37.8)$ & $6(16.2)$ & $8(21.6)$ & $3(8.1)$ & $6(16.2)$ \\
\hline Ability to process for radioactivity analysis & $15(40.5)$ & $6(16.2)$ & $7(18.9)$ & $3(8.1)$ & $6(16.2)$ \\
\hline Ability to ship for radioactivity analysis & $15(40.5)$ & $6(16.2)$ & $7(18.9)$ & $3(8.1)$ & $6(16.2)$ \\
\hline Ability to conduct radioactivity analysis & $15(40.5)$ & $7(18.9)$ & $6(16.2)$ & $3(8.1)$ & $6(16.2)$ \\
\hline \multicolumn{6}{|l|}{ Health assessment } \\
\hline Health physics interpretation of acute incident-radiation & $8(21.6)$ & $10(27.0)$ & $9(24.3)$ & $5(13.5)$ & $5(13.5)$ \\
\hline Health physics consultation on reentry-radiation & $7(18.9)$ & $11(29.7)$ & $11(29.7)$ & $4(10.8)$ & $4(10.8)$ \\
\hline Health physics predictions on long-term health effects-radiation & 7 (18.9) & $14(37.8)$ & $8(21.6)$ & $4(10.8)$ & $4(10.8)$ \\
\hline Medicine consults regarding radiation effects & $13(35.1)$ & $12(32.4)$ & $4(10.8)$ & $3(8.1)$ & $5(13.5)$ \\
\hline Early detection of radiation contamination in first responders & $6(16.2)$ & $5(13.5)$ & $17(45.9)$ & $7(18.9)$ & $2(5.4)$ \\
\hline
\end{tabular}

IT/GIS=information technology/geographic information systems. 
assessments, and other public health functions were reported as sufficient by $42 \%$ to $65 \%$ of states. These responses were among the strongest reported in this part of the assessment. Relationships with federal partners to handle biological/clinical samples were weaker $(33 \%-35 \%)$ for all 4 capabilities in this category.

\section{Overall Radiation Written Plan and Exercises}

Twenty (53\%) states reported having a finalized radiationspecific written response plan (Table 5). Four (20\%) of the 20 states did not have a nuclear power plant (data not shown). For unintentional releases, half of the states had written or detailed operations plans for all scenarios except for a waterways incident, for which only $6(15 \%)$ states reported having a written or detailed operations plan.
Half of the states reported having detailed operation plans for a radiologic contamination dispersal device, a so-called dirty bomb. For the intentional scenarios, approximately one-third of states $(31 \%-35 \%)$ reported having detailed operations plans.

Sixteen $(80 \%)$ of the 20 states with a written plan reported having conducted a drill or an exercise of the radiation plan. The most recent drill or exercise for these states (data not shown) occurred across the 4 time frames: within 6 months (6 [38\%] states), longer than 6 months ago but less than 12 months ago (3 [19\%]), 1 to 2 years ago (5 [31\%]), and longer than 2 years ago $(2[13 \%])$.

Twenty-one (57\%) states reported having mutual aid agreements for radiation emergency response with other states. Twenty-two $(60 \%)$ reported having these agreements with other

\section{TABLE 4}

\section{State Health Department Relationships Established With Federal Agencies for Radiation Emergencies}

\begin{tabular}{|c|c|c|c|c|}
\hline & $\begin{array}{l}\text { None, } \\
\text { No. }(\%)\end{array}$ & $\begin{array}{l}\text { Some, } \\
\text { No. }(\%)\end{array}$ & $\begin{array}{l}\text { Sufficient No. and } \\
\text { Level, No. }(\%)\end{array}$ & $\begin{array}{l}\text { Uncertain, } \\
\text { No. }(\%)\end{array}$ \\
\hline \multicolumn{5}{|l|}{ Epidemiology/surveillance: potential human effects of radiation } \\
\hline $\begin{array}{l}\text { Other kind of surveillance (eg, poison control center calls, pharmaceutical } \\
\text { purchases, school absenteeism), not included above }\end{array}$ & $10(26.3)$ & $15(39.5)$ & $8(21.1)$ & $5(13.2)$ \\
\hline Surveillance through astute health care providers' reporting & $12(32.4)$ & $8(21.6)$ & $10(27.0)$ & $7(18.9)$ \\
\hline $\begin{array}{l}\text { Crisis-phase epidemiology (eg, documenting acute morbidity, outbreak-style } \\
\text { investigation) }\end{array}$ & $10(27.0)$ & $10(27.0)$ & $10(27.0)$ & $7(18.9)$ \\
\hline \multicolumn{5}{|l|}{ Exposure assessment } \\
\hline Advice for radiation exposure assessment & $3(7.9)$ & $9(23.7)$ & $23(60.5)$ & $3(7.9)$ \\
\hline Advice regarding environmental sampling & $3(7.9)$ & $8(21.1)$ & $24(63.2)$ & $3(7.9)$ \\
\hline Advice regarding biological/clinical sampling & $2(5.4)$ & $13(35.1)$ & $18(48.6)$ & $4(10.8)$ \\
\hline Ability to conduct population-based exposure monitoring & $4(10.5)$ & $10(26.3)$ & $16(42.1)$ & $8(21.1)$ \\
\hline Ability to conduct radioactivity analysis & $1(2.7)$ & $9(24.3)$ & $21(56.8)$ & $6(16.2)$ \\
\hline \multicolumn{5}{|l|}{ For biological/clinical samples } \\
\hline Ability to collect for radioactivity analysis & $7(18.9)$ & $11(29.7)$ & $13(35.1)$ & $6(16.2)$ \\
\hline Ability to process for radioactivity analysis & $6(16.7)$ & $12(33.3)$ & $12(33.3)$ & $6(16.7)$ \\
\hline Ability to ship for radioactivity analysis & $7(18.9)$ & $11(29.7)$ & $13(35.1)$ & $6(16.2)$ \\
\hline Ability to conduct radioactivity analysis & $6(16.7)$ & $12(33.3)$ & $12(33.3)$ & $6(16.7)$ \\
\hline \multicolumn{5}{|l|}{ Health assessment } \\
\hline Health physics interpretation of acute incident-radiation & $1(2.6)$ & $8(21.1)$ & $24(63.2)$ & $5(13.2)$ \\
\hline Health physics consultation on reentry-radiation & $1(2.6)$ & $9(23.7)$ & $23(60.5)$ & $5(13.2)$ \\
\hline Health physics predictions on long-term health effects-radiation & $3(7.9)$ & $8(21.1)$ & $22(57.9)$ & $5(13.2)$ \\
\hline Medicine consults regarding radiation effects & $3(7.9)$ & $10(26.3)$ & $20(52.6)$ & $5(13.2)$ \\
\hline Early detection of radiation contamination in first responders & $4(10.5)$ & $10(26.3)$ & $17(44.7)$ & $7(18.4)$ \\
\hline IT/GIS integration of radiation exposure data & $5(13.2)$ & $7(18.4)$ & $17(44.7)$ & $9(23.7)$ \\
\hline
\end{tabular}

IT/GIS= information technology/geographic information systems. 
TABLE 5

\begin{tabular}{|c|c|c|}
\hline & \multicolumn{2}{|c|}{ Extent of Planning } \\
\hline Radiation Incident Type & $\begin{array}{c}\text { None or } \\
\text { Minimal, } \\
\text { No. (\%) }\end{array}$ & $\begin{array}{c}\text { Steps or } \\
\text { Detailed } \\
\text { Operations } \\
\text { Plan } \\
\text { Written, } \\
\text { No. (\%) }\end{array}$ \\
\hline Unintentional & & \\
\hline Transportation: roadway & $18(47)$ & $20(53)$ \\
\hline Transportation: waterway & $32(85)$ & $6(15)$ \\
\hline Hospital or medical & $17(45)$ & $21(55)$ \\
\hline $\begin{array}{l}\text { Fixed facility, not hospital or nuclear power plant } \\
\text { Intentional }\end{array}$ & $20(54)$ & $17(46)$ \\
\hline Mass gatherings (eg, Super Bowl) & $23(61)$ & $15(39)$ \\
\hline Major location (eg, capitol building) & $26(68)$ & $12(32)$ \\
\hline Explosive device, dispersal (ie, "dirty bomb") & $20(53)$ & $18(47)$ \\
\hline $\begin{array}{l}\text { Silent dispersal (ie, intentional radiation material } \\
\text { poisoning) }\end{array}$ & $25(66)$ & $13(34)$ \\
\hline $\begin{array}{l}\text { Nuclear detonation, including improvised } \\
\text { nuclear device }\end{array}$ & $26(68)$ & $12(32)$ \\
\hline
\end{tabular}

state agencies; $16(73 \%)$ of the 22 reported having drilled with their mutual aid partners. Thirty-seven $(97 \%)$ states reported having an on-call duty officer system that provides around-theclock coverage and prompt response to a radiation emergency incident.

\section{Radiation Emergency Staffing Levels in State Health Departments}

Thirty-eight states reported an average of 28 full-time staff members who would be available to respond to a large-scale radiation emergency incident. Of these, 22.8 (81\%) were public health agency employees working in other areas who would be assigned or redirected to response activities. The 26 states that have a nuclear power plant within their borders reported more than 4 times the available staffing to respond to a large-scale radiation emergency incident than the 12 states without a nuclear power plant (37 staff vs 9 staff). Nearly three-fourths (28) of the states reported that at least 1 of their local health departments had radiation emergency staff, and 23 (82\%) reported knowing either all (10 [27\%] states) or some (13 [34\%] states) of these staff members. Twenty-eight (74\%) states reported providing training to local jurisdictions on any aspect of radiation emergency preparedness and response.

\section{Interagency and Intra-agency Coordination}

The majority of states had coordinated with the following critical partners for a radiation emergency: state emergency management agency, state environmental health agency, state environmental agency, nuclear power plant, and National Guard Civil Support Team. At least 15 (40\%) states reported some level of coordination with their regional Federal Bureau of Investigation office, state food regulator, state environmental health agency, state environmental agency, poison control centers, and state emergency management services agency. At least $19(50 \%)$ states reported having actually conducted a tabletop or other exercise with partner agencies in the last 2 years. States reported less coordination with Native American/Alaska Native authorities (45\%), academic institutions (55\%), and state mental health departments (52\%).

Twenty-one (55\%) states reported that their teams meet with other parts of the health department. Of the 38 responding states, $11(29 \%)$ reported that the radiation response team is not part of the state health agency; in 19\% (5 of 26) of states with nuclear power plants the radiation response team was not located in the public health agency, whereas in 50\% (6 of 12) of states without nuclear power plants the radiation response team was not in the public health agency. Thus, states with nuclear power plants were more likely to have radiation response teams within the public health agency.

Of the 21 states that reported holding coordination meetings with other divisions of the state health agency, most reported meeting recently. Twelve (57\%) reported meeting within the last 6 months; $4(19 \%)$ states had met 6 to 12 months previously, and 5 (24\%) most recently met more than 1 year ago.

Nine (24\%) states reported having developed protocols for gathering epidemiologic and exposure data for a large radiologic incident that would involve more than 1 county. Most (25 [66\%]) states had no such protocols, and $4(11 \%)$ did not know. The responses for the 27 states with a nuclear power plant were similar to the aggregated total (20\%, yes; $65 \%$, no; $15 \%$, do not know). For the 12 states without a nuclear power plant, $4(33 \%)$ reported having established protocols for gathering epidemiologic and exposure data, and 8 (67\%) reported no such protocols.

Thirty-six states responded to the question about planning and response protocol for providing coordinated guidance for a largescale radiation emergency incident. Of those, $11(31 \%)$ states reported having such protocols, and 22 (61\%) did not; 3 (8\%) states did not know. Responses for the 24 states with a nuclear power plant were similar to the aggregated totals (33\%, yes; $58 \%$, no; $8 \%$, do not know). Of the 12 states without a nuclear power plant, 3 (25\%) reported having coordination protocols, 8 (67\%) states had no such protocols, and 1 (8\%) did not know.

\section{Overall Rating of Preparedness}

For the 38 states responding, the average subjective radiological preparedness score was 4.54 (on a scale of 1.0-10.0). Preparedness for the 26 states with a nuclear power plant was 4.76 and for the 12 without a nuclear power plant, 4.08. The average score for states that listed the state radiation control program director as a contributing respondent was $4.81(n=33)$ compared with states that did not $2.80(n=5)$. 


\section{DISCUSSION}

Although the results of the assessment are based on a response rate of $76 \%$ of state public health departments, findings can be generalized to the entire United States. Responding states were representative of the nation as a whole, given that respondents included geographically large and small states, all regions, dense and sparse populations, and states with presence and absence of nuclear power plants.

The results of this assessment indicate that in many measures of public health capacity and capability, the nation remains poorly prepared to respond adequately to a major radiation emergency incident. Capabilities are insufficient or inadequate throughout the functions assessing planning in state health departments, resources in the state health department and other state agencies, and relationships with federal and other partners. For some measures, as many as $85 \%$ of responding states reported insufficient capability to respond to a radiation incident.

The most fundamental step of preparedness, development of response plans (outside of response plans for nuclear power plant emergencies), was not reported as occurring in $45 \%$ of states. Without a comprehensive plan, states in which a radiation emergency occurs are likely to mount inefficient, ineffective, inappropriate, or tardy responses that could result in (preventable) loss of life. With nearly half of the responding states not having a response plan, a large portion of the US population is at increased risk should a radiological event occur within the country's borders.

Gaps were also evident related to specific aspects of public health preparedness. Few states reported having written protocols for epidemiologic or exposure data collection or for coordinated guidance. Without such guidance, the collection of information may be uncoordinated and important comparisons may be lost because critical information failed to be collected and analyzed. Delays in collection may affect data quality.

Despite long-standing awareness of the threat of unintentional releases of radioactivity within our communities, in general, less than half of the states reported having a written plan or detailed plan of operations for responding to releases, with the exception of plans for releases associated with roadway incidents. Preparedness was even less developed with regard to intentional releases. The exception to this gap in operations planning was for response to a radioactive dispersal device, for which nearly half of the states have written or detailed operations plans. The large number of states reporting a plan in response to a radioactive dispersal device may reflect the interest and support of federal funding for terrorism that included dispersal devices as the prototypic radiation threat in US communities.

The results of this survey suggested that public health agency staffing to respond to a radiation emergency incident would rely heavily on redirecting state staff from their regular assignments to radiation emergency response activities. Although this survey did not inquire about the training of such surge staff, this finding suggests that states should attend to the training needs of these personnel.

The lack of a sizable cadre of radiation emergency workers is further highlighted when the states with and without nuclear power plants are separated. States with nuclear power plants estimate having 4 times as many staff available to them, either directly or through redirection, than do states without such plants. This capacity may result from long-term direction and assistance from state and federal agencies that regulate nuclear power plants. Even though having a nuclear power plant within a state increases the risk for a radiation emergency and thus the need for response capacity, radiation emergencies not involving power plants are possible everywhere, and the lack of adequate response personnel in many states is of concern.

The limitations of this survey include an incomplete response rate $(76 \%)$ and incomplete data provided on some questions. In addition, states used a range and variety of individuals to complete the survey, and some states may not have reached out to all of the appropriate individuals, resulting in responses that may not have been correct. A number of states were not sure of partnerships or agreements that were in place, indicating that not all of the questions were answered by the most knowledgeable staff member. Also, some survey questions may have been interpreted differently among the states.

Nevertheless, acknowledging these limitations, the results from this assessment highlight the many aspects of radiation emergency response for which states are not adequately prepared. In addition, they support the findings of earlier assessments, including the 2003 CSTE survey $^{1}$ and the 2010 public health laboratory capabilities assessment. ${ }^{2}$ The CDC has undertaken a number of activities to improve on this situation, including the development of training materials $s^{3}$ and the formation of NARR, which is an important step toward improved coordination of public health's role in radiation emergency response. Based on the findings of this report, a number of recommendations are appropriate, including the following activities:

- Collect and disseminate best practices in state-based radiation response plans (excluding nuclear power plant plans) in coordination with CSTE's "disaster epidemiology"4 workgroup in the development of plans for radiation exposure public health surveillance.

- Explore with CDC how best to incorporate radiation preparedness as a priority under the new all-hazards guidance and appropriate capabilities development.

- Substantially increase training in radiation emergency response for public health personnel, especially personnel without related expertise who would be called upon to provide services during a widespread radiation emergency.

- Conduct strategic planning for activities that will increase collaboration between state public health personnel in CDC- 
funded preparedness and radiation protection personnel, including health physicists, radiation equipment licensing, and inspection personnel, who are often in the regulatory, rather than the public health, area of health departments or who may not be part of the health department at all.

- Develop exercise templates for radiation release scenarios (non-nuclear power plant related) that would apply at the state and local health department levels (ie, not huge but still with a large impact).

- Coordinate with the Office of the Assistant Secretary for Preparedness and Response (ASPR) at its funded state health department programs and the federal level on aspects of radiation preparedness at the state and local levels that involve medical treatment, hospital surge, and so forth.

- Encourage local and state health departments to establish collaborations and connections with state, regional, and federal response partners who are versed in responding to incidents involving radiation (eg, the Civil Support Team of the state National Guard, regional response teams, Federal Emergency Management Agency, and Department of Energy radiation response teams).

Author Affiliations: Dr Watkins is with the Florida Department of Health, Division of Environmental Health; Dr Perrotta is with the Texas A\&M Health Science Center; Ms Stanbury is with the Michigan Department of Community Health, Division of Environmental Health; Mr Heumann is with the Oregon Public Health Division; Dr Anderson is with the Wisconsin Division of Public Health; and Ms Simms and Ms Huang are with the Council of State and Territorial Epidemiologists.

Correspondence: Address correspondence and reprint requests to Dr Sharon Watkins, Senior Environmental Epidemiologist, Florida Department of Health, 4052 Bald Cypress Way, Bin A08, Tallahassee, FL 32399 (e-mail: Sharon _Watkins@doh.state.fl.us).
Received for publication January 6, 2011; accepted January 18, 2011.

The CSTE conducted this assessment with financial support from the Association of State and Territorial Health Officials and the CDC (cooperative agreement $1 \mathrm{U} 38 \mathrm{HM}$ 000454). The views and opinions expressed in this document are solely those of the CSTE Workgroup, unless otherwise indicated, and may not necessarily represent the views and opinions of the member associations of NARR or the CDC. Although the information in this document may be used by the CDC to develop effective public health guidance and guide future actions, any statements made or actions taken in response to this report do not necessarily constitute endorsement or agreement by the CDC or NARR of the findings, conclusions, and recommendations contained in this article.

Author Disclosures: The authors report no conflicts of interest.

Acknowledgments: CSTE thanks the Radiation Emergency Workgroup members for offering their time and expertise in drafting this assessment. Representatives from state health departments, the Conference of Radiation Control Program Directors, the Association of State and Territorial Health Officials, the National Association of County and City Health Officials, and the CDC collaborated on this project. CSTE greatly appreciates and acknowledges the thoughtful input provided by all respondents.

\section{REFERENCES}

1. Council of State and Territorial Epidemiologists. A national assessment of the status of planning for public health preparedness for chemical and radiological contaminating terrorism: CSTE's findings and recommendations. http: //www.cste.org/dnn/LinkClick.aspx?fileticket=vtcs94oAGYE\%3d\&tabid $=175 \& \mathrm{mid}=716$. http: $/ /$ www.cste.org $/$ webpdfs $/ 2010$ raditionreport.pdf Published 2004. Accessed September 12, 2010.

2. Association of Public Health Laboratories. APHL All-Hazards Laboratory Preparedness Survey data. 2009. http://www.aphl.org/aphlprograms/phpr /ahr/Documents/DiminishingResourcesEvolvingThreats.pdf. Accessed February 21, 2011.

3. Centers for Disease Control and Prevention (CDC). CDC Grand Rounds: radiological and nuclear preparedness. MMWR Morb Mortal Wkly Rep. 2010;59(36):1178-1181.

4. Council of State and Territorial Epidemiologists. Disaster Epi workshop. http://www.cste.org/dnn/ProgramsandActivities/DisasterEpiWorkshop/tabid /404/Default.aspx. Accessed October 25, 2010. 